\title{
Card Fraud Reduction Technology in Biometric Fingerprint Security Using Data Mining Algorithms
}

\author{
P.Gayathiri, M. Punithavalli
}

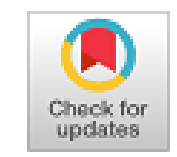

\begin{abstract}
In the banking sector, biometric authentication is a new trend of technology for avoiding card fraud in the card payment system. A major part of the failure of the partial fingerprint happens to internet banking, mobile banking, e-commerce, Point of scale, and ATM. The partial fingerprint or incomplete fingerprint is a poor quality of ridges in minutiae, noise, and absence of minutiae features. To overcome this problem, many researchers are investigated and find the solution to partial fingerprint techniques like enrollment process, verification, identification and classification of existing algorithms. In the acquisition, process sensors capture the fingerprint image using an optical scanner. A fingerprint-based biometric system is largely a pattern popularity device that allows $s$ someone through the authenticity of the fingerprint-based on biometric verification and identity system. The verification system Acquisition process of fingerprint image can be Compared to database image verify the person is authorized or not. The identification process recognizes the person by one-to-many verification. this paper detailed study of the partial fingerprint techniques time complexity of the Data Mining Algorithms.

Keywords : Biometric, Card Payment, Data mining,Fingerprint
\end{abstract}

\section{INTRODUCTION}

Biometric in banking is more reliable and secure in the biometric authentication system, Biometric characteristics of the physical and behavioral is a unit of measurement distinctive for each individual on the world and extremely sturdy to spoof or forge that is why the technology is commonplace in high-security government operations like border management, jail management, and national ID. Traditional methods of passwords and PIN identification are given less security. Nowadays-business is coming up with new fingerprint readers based totally on newer technology each day to make fingerprint identification process less difficult. The USB fingerprint readers are ready with optical sensors which might be vibration and can sense the touch of a finger in its area. The advanced technology guarantees that below any situation it may take a clear and distortion-free image of a finger regardless of whether or not wet or even aged[1].

Revised Manuscript Received on October 30, 2019.

* Correspondence Author

P.Gayathiri Department of Computer Applications, Bharathiar University, Coimbatore,India Email: gai3teju2017@gmail.com

Dr. M. Punithavalli, Department of Computer Applications, Bharathiar University, Coimbatore,India

(C) The Authors. Published by Blue Eyes Intelligence Engineering and Sciences Publication (BEIESP). This is an open access article under the CC BY-NC-ND license (http://creativecommons.org/licenses/by-nc-nd/4.0/)

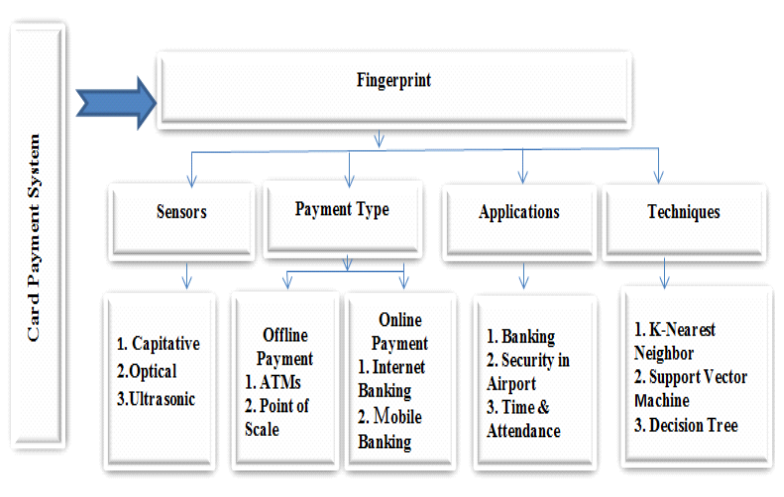

Figure 1 for Architecture of Card Payment System

\section{A. Applications Of Biometrics In E-Banking}

1) Biometrics for Online Banking: Banking establishments are currently giving fingerprint verification through the mouse that the A customer will safely access the internet banking shown in figure 4 architecture of the card payment system.

2) Biometrics in ATMs: There are 2 approaches to using Biometric Authentication in ATMs.

3) Biometrics for Mobile Banking: In several smartphones bank offers money transactions and customer service through voice or speech recognition[2].

\section{B. Data Mining}

Data Mining is to extract the features from a large dataset and particular class to be assign. It is used to extract features in data classes within the given data set[3]. A classification describes in two methods. First Method is to classify the training data set in classification algorithm and the second method is to test the data in extracted features to measure performance and accuracy. Merchants mostly agree that automating knowledge analysis to misbehavior. Machine learning will hunt down customers World Health Organization unusually behaves with a merchant[4]. This technology will offer suspicion scores, rules, and visual unusual person. This could be done that makes it an excellent fraud prevention solution.

\section{FINGERPRINT RECOGNITION}

Fingerprint uniquely to identify the person for the representation of the ridge and furrows. A fingerprint is comprised of ridges and valley. 


\section{Card Fraud Reduction Technology in Biometric Fingerprint Security Using Data Mining Algorithms}

The ridges floor area building blocks the dark endless of the fingerprint and additionally the valleys area white area that exists among the ridges. Several classifications place units have been given to traffic patterns to get up inside the ridges known as the minutiae of the fingerprint. Fingerprint features of ridge ending and bifurcation as shown in figure 2 .

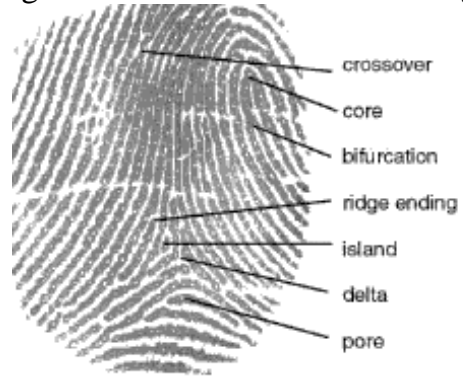

Figure 2 fingerprint features source[12]

Fingerprint recognition consists of taking a fingerprint image of someone and records its capabilities like arches, whorls, and loops shown in figure 3 Matching of the Fingerprint can be minutiae, correlation, and ridge based matching[5].
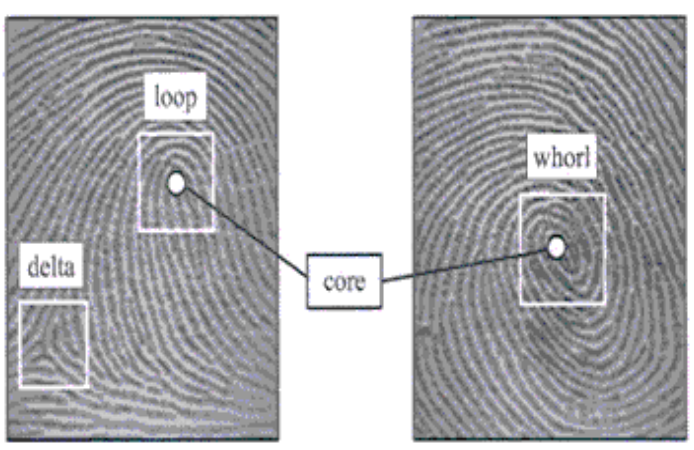

\section{Figure 3 for fingerprint patterns}

- Minutiae-based total fingerprint matching stores an image in fixed of points and therefore the set of points are corresponding inside the template and therefore the enter minutiae. Correlation-based fingerprint matching overlays fingerprint snapshots and association among equal pixels is calculated. Ridge characteristic primarily based fingerprint matching is an associate degree revolutionary technique that captures ridges, as trivialities-based fingerprint capturing of the fingerprint image[6]. Fingerprints are fairly dependable of identification because of their individuation and Fingerprint process split into an enrollment module, Verification, and identification shown in figure 6.

\section{LITERATURE REVIEW}

ShengdaHu[1] proposed partial fingerprint recognition on deep learning algorithms. It can improve the structure of convolutional neural networks, The contribution of work is especially as follows: one. a way of extracting features partial fingerprint image employing a residual network. Train the designed residual network using Cross-Entropy to perform and Contrast-Loss function so get the stable feature by k-means++ algorithm.Nadja et al [3] planned implementation square measure supported the Skin snap Tolerant algorithmic program (SETA), that uses minutiae to implement fingerprints matching consist of alignment between the fingerprints. Although economical SETA is not acceptable for card implementation as a result of it desires lots of dynamic storage memory to store the counters of all potential rotations and translations.

ZhangJie[13] presented Incomplete fingerprint recognition using pattern entropy-based similarity measure. The global orientation and local minutiae features are extracted and merged to construct a unique scale and rotation invariant feature. The pattern entropy for similarity measure that eliminates most of the false matches and performs time complexity

Kai Cao[14] proposed a novel fingerprint classification algorithm orientation model is to improve the fingerprint extraction using Complex filter fingerprint ridge and singularities. A heuristic classifier that's powerful to intra-classification variability's and inter-category variability's to gain a higher accuracy.

Tsai-Yang Jea[23] presented a new minutiae-based partial fingerprint matching using Support Vector Machine (SVM). They have designed an SVM to classify the partial fingerprint with the input (query) and (registered) fingerprints square measure at the beginning matched per their minutiae points challenges of minutiae-based partial fingerprint matching square measure

- The problem is poor quality fingerprints.

- They need for additional features (than minutiae) to improve the accuracy.

- Global minutiae matching square measure neither robust nor efficient to handling distortion. Jing-Ming Guo[11] proposed a rule-headquartered fingerprint classification method to center-to-Delta float (CDF) and balance Arm waft (BAF) rectangular measure. In classification methods, the two key issues impact the accuracy is the normal of a fingerprint image and the anomaly of a classification.

WonjuneLee[6] developed the Partial Fingerprint Matching process of ridge form points (RSFs) the small ridge segments Within the minutiae matching degree corresponding minutiae pairs are decided by way of evaluating the nearby RSFs and adjoining to every minutia. During the subsequent ridge-operate-matching stage, the RSFs within the overlapped pix are extra in assessment to embellish the matching accuracy.

Paul W.H[10] Proposed shape context descriptor for point pattern matching. Modified matching form contexts utilizing specified contextual information can give a boost to the fingerprint matching when compared with the original image. To minimize computation in the pre-processing step termed elliptical region filtering is unromantic in putting off spurious minutiae before matching.

S. Milstein et al. [25] proposed a fingerprint attention algorithm for partial and entire fingerprints. The Space Frequency Transformation Algorithm (SFTA) and Line Scan Algorithm (LSA) developed to examine partial fingerprints and diminish the time taken to assess full fingerprints. LSA gives a thoroughly green reputation procedure. Essentially the most advantages of those algorithms core factor given the excessive accuracy in partial fingerprints. At this time the important drawback of evolved algorithms is the lack of pre-form of validated palms. 
RuZhou[12] proposed Fingerprint identification the use of SIFT-based trivialities Descriptors and the overall performance of trivia primarily based fingerprint authentication algorithms degrades drastically even as handling low best fingerprints with cuts can overcome this issues using Scale Invariant characteristic Transformation (SIFT) descriptors the use of SMD and IADM (FISA) finished and given better accuracy algorithm to compared with the existing algorithms.

HelalaAlShehri[5] proposes a singular fingerprint verification technique to resolve the trouble of move-matching. Fingerprints are captured from the equal finger using different sensors they range in scales and determination. To those troubles, the alternatives of a fingerprint are regionally extracted from it by using decomposing the fingerprint into overlapping home windows. Binary patterns (LBP) and Scale Invariant Feature Transformation (SIFT) Descriptors using local ridge patterns. Descriptors can produce every rotation-invariant and scale-invariant LBP represent the vital systems of the fingerprints like edges SIFT has moreover been verified the descriptor due to the fact it is scale-invariant and rotation invariant.

Tsai Yang Jea[23] proposed partial fingerprints in the fingerprint identification process. There is additionally considerable hobby in process partial and latent fingerprints acquired at crime scenes. Once the partial print would not embrace structures like center and delta, matching strategies supported the alignment of singular structures fail. The overlapping regions minutiae matched with the two-hidden-layer Network.

AbinandhanChandrasekaran[26] developed a fingerprint matching using a tree structure. The minutiae point from the template and enter fingerprint image had been acquired by way of the use of ratios of relative distance because of the evaluating function. Bottom-up method connecting the minutiae points and separated the spurious and then calculated a matching rating. The matching score was acquired with the aid of evaluating the similarity of the 2 tree structures primarily based on a threshold cost.

Israa Ghazi Dakhil[2] proposed the design and Implementation of the Fingerprint identification system based totally on the KNN Neural network using the Filterbank Based method. KNN Neural networks provide the perfect matching result and the threshold approach to provide suitable and exact termination for fingerprint accuracy is 93.9 $\%$.

Sunil Kumar Singla[16] proposed the 2012 Genetic Algorithm based relative alignment method has been proposed yet implemented the translational then rotational of fingerprint images. All parameters $\mathrm{x}, \mathrm{y}$ (translational), and(rotational) hold been optimized separately or the fitness value has been calculated using the parameters beside reference and template images can speed up the process using Binary Search Algorithm. If the measure regarding tolerance is improved to five pixels and two rate accuracy will become $98 \%$.

Paul W.H. Kwan[10] developed the shape context descriptor using fingerprint matching. The expense improves coordinating precision when contrasted with the first picture. To diminish calculation circular area separating is proposed for expelling misleading details preceding coordinating. Tests affirmed the enhancements in precision and speed achieved by the proposed technique.
Muhammad TalalIbrahim[17] proposed Fingerprint Verification Using Rotation Invariant Feature Codes in the picture based unique finger impression check framework. Improves an information unique mark picture utilizing a complex separating strategy of the recurrence area or utilize the intricate fillers to recognize the center point. Accordingly, a locale of intrigue (ROI) over a predefined measure, which revolves around the recognized center point, is separated. The subsequent ROI is surrounded fundamentally based in regards to the identified center consider edge similarity with guarantee revolving around invariance. The proposed technique extricates the Gabor channels are connected to the ROI. To limit the dimensionality over the separated applications even as delivering the additional biased representation. $T$ his paper thinks about the unsupervised Principal Component Analysis and the directed Linear Discriminant Analysis methods in light of dimensionality reduction.

AneeshaKarar[8] proposed Fingerprint Enhancement using Thinning Minutiae Extraction regarding Ridge or Bifurcation. the enhancement of a fingerprint using the anisotropic filter. The contribution of the work is fingerprint recognition by extracting the minutiae using the thinning algorithm. The result gives higher performance accuracy.

AmjadAli[9] proposed Minutiae based Automatic Fingerprint Recognition using Machine Learning Algorithms classified by fingerprint recognition. Minutiae features in the surrounding region core of fingerprint images. ThePoint Index technique is utilized in determining the core point followed by the enhancement using a Gabor filter. The consequences exhibit that Random Forest then Radial Basis Functions hand over better outcomes for various quality images in comparison to other existing algorithms.

Kumarattangudiperichiappan[5] developed fingerprint recognition using minutiae extraction. Generally utilized subprogram for enhancing fingerprint simulacrum quality using the Fourier spectrum. For efficient matching and feature extraction algorithms, As a pre-processing method, we want to carry out comprising of discipline introduction, ridge frequency estimation, Sobel filtering, department, and thinning

algorithm. 
Table- I:Limitations of Existing Algorithms

\begin{tabular}{|c|c|c|c|}
\hline S.NO & Author/Year & Methods & Demerits \\
\hline 1 & Fang Zang (2018) & CNN & $\begin{array}{l}\text { Feature extraction and minutiae } \\
\text { matching is the most time-consuming } \\
\text { in a large database }\end{array}$ \\
\hline 2 & Parvindar Singh (2016) & Hybrid Shape Orientation Descriptor Algorithm & Less computation time \\
\hline 3 & A.E Amin (2016) & Shape Matching Algorithm & $\begin{array}{l}\text { Reduces computational complexity by } \\
\text { significantly improve accuracy and } \\
\text { speed }\end{array}$ \\
\hline 3 & Xiang fu( 2015) & Minutiae tensor Matrix Spectral Matching & $\begin{array}{l}\text { Minutiae Pair that exhibit low } \\
\text { similarities and comp abilities remain } \\
\text { unsolved }\end{array}$ \\
\hline 4 & Pablo David (2014) & Minutiae cylinder code & $\begin{array}{l}\text { Less running time obvious that both } \\
\text { feature extract and similarity measure } \\
\text { and lowest accuracy }\end{array}$ \\
\hline 5 & $\begin{array}{l}\text { Ru Zhou's } \\
\text { (2013) }\end{array}$ & Akaze & $\begin{array}{l}\text { it does not perform low quality of } \\
\text { images. }\end{array}$ \\
\hline 6 & Cantarella(2013) & SIFT Local Minutiae Matching & Distortion in image acquisition \\
\hline \multirow[t]{2}{*}{7} & AttaneChatterjee ( 2010) & ANN & $\begin{array}{l}\text { Slow learning speed and poor learning } \\
\text { scalability }\end{array}$ \\
\hline & Chikkeru\&Govindha(2010) & Chain code & $\begin{array}{l}\text { Accuracy degrades significantly with } \\
\text { an inadequate number of minutiae }\end{array}$ \\
\hline 8 & Weiguo sheng( 2007) & Genetic Algorithm & $\begin{array}{l}\text { a local improvement operator was used } \\
\text { to effectively design an efficient } \\
\text { algorithm for the minutiae point } \\
\text { pattern matching problem. }\end{array}$ \\
\hline 9 & JianjiangFeng 2008 & Support Vector Machine & $\begin{array}{l}\text { minutiae template does not contain } \\
\text { sufficient } \\
\text { information to allow the reconstruction } \\
\text { of the original } \\
\text { fingerprint image. }\end{array}$ \\
\hline 10 & Tsai Yuang 2005 & ANN & $\begin{array}{l}\text { More noise in minutiae and less } \\
\text { complexity }\end{array}$ \\
\hline 11 & JeaGovindaraj 2005 & Correlation features & $\begin{array}{l}\text { Correlation Features performs not well } \\
\text { in low-quality images with noise and } \\
\text { distortion }\end{array}$ \\
\hline
\end{tabular}

\section{RESULT AND DISCUSSION}

The review of the paper discussed the partial fingerprint authentication for internet banking, mobile banking, e-commerce, Point of scale and ATM for card payment is additionally reliable and secure. Fingerprint authentication of a partial fingerprint image has the smallest number of minutiae are twenty to the thirty. The overall analysis of partial fingerprint techniques is mentioned within the enrollment method, verification, identification and classification algorithms accuracy and time quality of algorithms are discussed.

\section{REFERENCES}

1. Shengda Hu partial fingerprint recognition algorithmic rule-based on deep learning for the recognition of partial fingerprint images S.I. Emergence in Human-like Intelligence towards Cyber-Physical Systems First Online: 30 June 2018.

2. Israa Ghazi Dakhil, Ali Abdulhafidh Ibrahim 2018 proposed Design, and Implementation of Fingerprint Identification System Based on KNN Neural Network, Journal of Computer and Communications, 2018, 6, 1- 18http://www.scirp.org/journal/jccISSN Online: 2327-5227ISSN Print: 2327-5219

3. NadiaNedjaha, Rafael.Wyant, Luiza.Mourelle Efficient Fingerprint Matching on Smart Cards for High Security and Privacy in Smart Systems Future Generation Computer Systems Volume 76, November 2017, Pages 18-32

4. Hella AlShehri1, Muhammad Hussain 2018 propose a novel fingerprint verification method to solve the problem of cross-matching Expert Systems With Applications Received date: 31 January 2017 Revised date: 1 June 2017 Accepted date: 12 June 2017

5. perichiappan 2017 proposed accurate fingerprint enhancement and identify the usage of minutiae extraction, Journal of Computer and Communications, 2017, 5, 28-38http://www.scirp.org/journal/jccISSN Online: 2327-5227ISSN Print: 2327-5219

6. Wonjune Lee, Sungchul Choa Proposed Partial Fingerprint Matching Using Minutiae and Ridge Shape Features for Small Fingerprint Scanners, Expert Systems With Applications 31 January 20171 June 201712 June 2017
7. Costas, Andrea, Boult, 2016 the partial fingerprint matching using the SIFT features FINAL, AUGUST 2016

8. Aneesha Karar, Prof.Amarjeet Kaur proposed Fingerprint Enhancement then Feature Extraction using Thinning then Minutiae Extraction regarding Ridge or Bifurcation, International Journal of Advanced Research in Computer Engineering \& Technology(IJARCET)Volume 4 Issue 6, June 2015

9. Amjad Ali1, Rehanullah Khan proposed Minutiae based Automatic Fingerprint Recognition, Published in 2015 IEEE International Conference on Computer and Information Technology; Ubiquitous Computing and Communications; Dependable, Autonomic and Secure Computing; Pervasive Intelligence and Computing IEEE Xplore: 28 December 2015

10. Paul W.H. Kwan1, Junbin Gao2 2015 proposed Shape context, a robust descriptor for point pattern matching, Pattern Recognition $\begin{array}{llllll}\text { Volume } & 47 & \text { Issue } & 3, & \text { March } & 2014\end{array}$ Pages 1469-1484

11. Jing-Ming Guo a, Yun-Fu Liu a Fingerprint classification based on decision tree from singular points and orientation field, Expert Systems with Applications 41 (2014) 752-764

12. Ru Zhou 1, Dexing Zhong proposed Fingerprint Identification Using SIFT-Based Minutiae Descriptors and Improved All Descriptor-Pair Matching, Sensors 2013, 13, 3142-3156;

13. .ZHANG Jie, JING Xiao-jun 2013 proposed Incomplete fingerprint recognition based on feature fusion and pattern entropy, Dianzi Yu Xinxi Xuebao/Journal of Electronics and Information Technology 34(12):3040-3045 - December 2013

14. Kai Cao a , Liaojun Pang a , Jimin Liang2013 a proposed Fingerprint classification by a hierarchical classifier attern Recognition archive Volume $46 \quad$ Issue 12, December, 2013 Pages 3186-3197

15. O. Arakawa, Adekunle Ajasin 2012 proposed Enhanced Biometric Authentication System for Efficient and Reliable e-Payment System in Nigeria. international Journal of Applied Information SystemsYear of Publication 2012

16. Sunil Kumar Singla1and Ajat Shatru Arora proposed 2012 Optimizing The Rotation And Translation Of Fingerprint images Using Genetic Algorithm, Applied Artificial Intelligence 26(6):541-553 - July 2012

Published By:

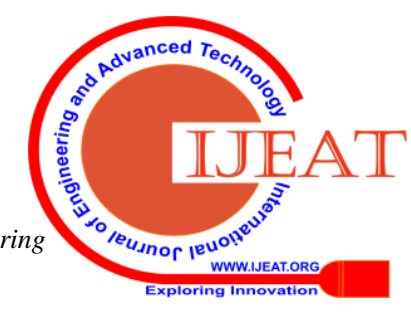


17. Muhammad Talal Ibrahim, Yongjin Wan proposed Fingerprint Verification Using Rotation Invariant Feature Codes. Digital Signal Processing Volume 20, Issue 4, July 2010, Pages 1264-1273

18. Jinjiang Feng 2008 descriptor-based minutiae matching algorithm, Pattern Recognition Volume 41, Issue 1, January 2008, Pages 342-352

19. Faraj Alhwarin, Chao Wang, Danijela Risti -Durrant, Axel Gräser, Improved SIFT-Features Matching for Object Recognition", BCS International Academic Conference 2008 -Visions of Computer Science

20. . M. Bicego, A. Lagorio, E. Grosso, and M. Tistarelli, On the Use of SIFT, Features for Face Authentication, Computer Vision and Pattern Recognition Workshop (CVPRW'06), 35, 2006.

21. . D. R. Kisku, A. Rattan, E. Grosso, and M. Tistarelli, Face Identification by SIFT-based Complete Graph Topology, Automatic Identification Advanced Technologies, 63-68, 2007.

22. Paul W.H. Kwan1, Junbin Gao2, and Yi Guo1 2006 Proposed Fingerprint Matching using Enhanced Shape Context. Article . January 2006

23. Tsai-Yang Jea, Venu Govindaraju 2010 presented a new minutiae-based partial fingerprint matching using Support Vector Machine (SVM) Pattern Recognition Archive Volume 38 Issue 10, October 2005 Pages 1672-1684

24. Davide Malton A Tutorial on Fingerprint Recognition, Biometric Systems Laboratory - DEIS - University of Bologna via Sacchi 3, 47023,

25. D. Maltoni, D. Maio, A.K. Jain, S. Prabhakar, Handbook of Fingerprint Recognition, Springer-Verlag, 2003.

26 . Abinandhan Chandrasekaran; Bhavani Thuraisingham Fingerprint Matching Algorithm Based on Tree Comparison using Ratios of Relational Distances, The Second International Conference on Availability, Reliability, and Security (ARES'07) 2007

\section{AUTHORS PROFILE}

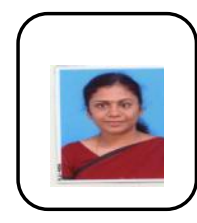

P.Gayathiri is pursuing as an Ph.D in the Department of Computer Applications at Bharathiar University, Coimbatore. She pursued her Bachelor of Computer Science at Madras University, Master of Computer Science at Krishna college of arts and science, under Madras University and. Her area of specialization is Data mining and Image processing

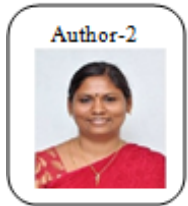

Dr. M. Punithavalli is working as an Associate Professor in the Department of Computer Applications at Bharathiar University, Coimbatore. She pursued her Bachelor of Computer Science at Dr. G.R. Damodaran College of Science, Coimbatore, Master of Computer Science at Avinashilingam University, M.Phil at Sri Vasavi College, Erode, under Bharathiar University and Doctor of Philosophy at Alagappa University, Karaikudi, on 2007. Her area of specialization is Data mining. 